Pak. j. sci. ind. res. Ser. A: phys. sci. 2017 60(2) 59-66

\title{
Isolation and Identification of Catechin by a New Method from Food Efficiency Stimulating Plant Alhagi camelorum
}

\author{
Abdul Hafeez Laghari ${ }^{a *}$, Shahabuddin Memon ${ }^{\mathrm{b}}$, Aisha Nelofar ${ }^{\mathrm{a}}$ and Khalid Mohammad. Khan \\ ${ }^{a}$ PCSIR Laboratories Complex, Karachi-75280, Pakistan \\ ${ }^{b}$ National Center of Excellence in Analytical Chemistry, University of Sindh, \\ Jamshoro 76080, Pakistan \\ ${ }^{c}$ H. E. J. Research Institute of Chemistry, International Center for Chemical and Biological Sciences, \\ University of Karachi, Karachi-75270, Pakistan
}

(received October 18, 2016; revised February 27, 2017; accepted March 9, 2017)

\begin{abstract}
The present article describes a new developed method for the simultaneous determination of (+)-catechin (1) and (-)-epicatechin (2), separated via HPLC. The method has been validated and applied on the real samples and $\mathbf{1}$ has been detected in both aerial and root parts of Alhagi camelorum without any ambiguity of fake positive or negative presence of $\mathbf{1}$ or $\mathbf{2}$ by virtue of dual detection system of UV and Mass Spectrometry. The antioxidant capacity was also investigated and a linear correlation has been noticed between the antioxidant capacity and the catechin amount in A. camelorum extracts
\end{abstract}

Keywords: catechin, Alhagi camelorum, HPLC, isolation

\section{Introduction}

Plants and their extracts have largely been analysed to determine active components, which make them suitable for pharmaceutical industries (Terao et al., 1994). (+)catechin (1) and (-)-epicatechin (2) are main representatives of one of six flavonoid classes widely analysed by different methods in various samples. They themselves as well as their derivatives are found to be active in curing many diseases. Their antioxidant and free radical scavenger activities are most important (Mendoza-Wilson and Glossman-Mitnik, 2006; Wolfe et al., 2003). Different researchers have stressed their role in reducing tumour development and growth (Okabe et al., 1999; Gali et al., 1994). They have ability to inhibit platelet aggregation and show antibacterial and angio-protective properties (Chang and Hsu, 1989). Normalization of blood pressure, prevention of endothelial dysfunction, insulin resistance in prediabetes stage as well as contribution to beneficial effects on the vascular system has also been attributed to catechins intake (Ihm et al., 2009). Their ability to induce selectively Phase I and II metabolic enzymes makes them an important class of drugs (Sohn et al., 1994; Vennat et al., 1988). Besides these activities, effect of catechins on metabolism has also been reported in different studies (Crespy et al., 2003; Donovan et al., 2001). Silberberg et al. (2005) have studied the effect

*Author for correspondence; E-mail: hafeezlaghari58@yahoo.com of catechin on absorption and metabolism in rats with co-administration of quercetin and found significant results. They have also recommended and verified that high nutritional intake of different flavonoids may significantly affect their respective absorption and metabolism. Nevertheless, correlation of food efficiency stimulating property of Alhagi camelorum extracts has still been ambiguous. Though it is difficult to quantify the consumption of foods and beverages, but estimation of constituents like catechins in this case may lead to know the actual reason of increased food intake (Auger et al., 2004).

Catechin has been found in different plants, likewise it has also been reported that the extracts of $A$. camelorum contain catechin in a quantifiable amount (Teissedre and Landrault, 2000). Extracts of $A$. camelorum have been reported to have different pharmacological activities (Marashdah and AL-Hazimi, 2010) whereas isolation of bio-molecules has also been done. A. camelorum has also been reported to have food efficiency stimulating property (Naseri and Mard, 2007) and the presence of flavonoid in $A$. camelorum has already been reported (Shaker et al., 2010; Naseri and Mard, 2007). The present study was based on the thought that not only 1 but both compounds (1) and (2) may be present in $A$. camelorum, which are responsible for the above mentioned activities. It was also aimed to develop and validate new and versatile method for the analysis of 
(1) and (2) in plant extracts in shorter time with more accuracy and precision.

\section{Materials and Methods}

Reference standards of $(+)$-catechin and (-)-epicatechin were purchased from Sigma Aldrich (St. Louis, MO, USA). Acetonitrile, methanol, formic acid, solid phase cartridge were purchased from Merck (Darmstadt, Germany). Butylhydroxyanisole (BHA) was purchased from BDH Chemicals Ltd (Poole, England). Deionized water was supplied by PCSIR Laboratories. Ethanol purchased from various sugar industries and purified in PCSIR Laboratories was of reagent grade. Raw material of $A$. camelorum was prepared from plant which was collected from vicinity of village Malkani located in district Badin of Sindh province of Pakistan, in February 2010. It was identified in Institute of Plant Sciences, University of Sindh, Jamshoro where a voucher specimen is deposited (A. camelorum 15460 ).

Preparation of standards. $25 \mathrm{mg}$ of each (1) and (2) were weighed and transferred to $25 \mathrm{~mL}$ volumetric flask containing $15 \mathrm{~mL}$ of methanol. Flasks were shaken and made up to volume by methanol to have $1000 \mathrm{mg} / \mathrm{L}$ (stock solution). $0.25 \mathrm{~mL}$ from each solution was taken and transferred to a $10 \mathrm{~mL}$ volumetric flask. Volume was made up by methanol in order to make $5 \mathrm{mg}^{2} / \mathrm{L}$ concentration of that mixture. From this mixture further dilutions were made in the range of $1.5-25 \mathrm{mg} / \mathrm{L}$.

Extraction procedures. Three different extraction techniques were applied to get extracts from plant material. Root parts were labelled as $\mathrm{R}$ and aerial parts as $\mathrm{A}$, while extraction techniques were abbreviated as (Son), (MW) and (Vor) for sonication, microwave and vortex mixing, respectively. Previously dried and powdered plant material ( $0.2 \mathrm{~g})$ was taken in each technique. In these techniques two extracting solvent systems were used (Iacopini et al., 2008) i.e., S1 or S2 comprising of $0.12 \mathrm{M}$ solution of hydrochloric acid in methanol:water or ethanol:water system $(7: 3 \mathrm{v} / \mathrm{v})$, respectively.

Microwave extraction. Samples of aerial and/or root parts of A. camelorum were transferred to pressure controlled Teflon vessels of the Start E Microwave extraction system (C) 2003 Mile Stone Inc. in triplicate followed by the addition of $10 \mathrm{~mL}$ of each solvent system S1 or S2. The programming of microwave extraction system was set in two steps. In the first step, gradient rise of temperature was maintained from ambient to $45^{\circ} \mathrm{C}$ within $5 \mathrm{~min}$ and in the $2^{\text {nd }}$ step it was kept constant for 15 min using an energy level of 400 $\mathrm{W}$, followed by ventilation for $10 \mathrm{~min}$.

Sonication. Samples were placed in $50 \mathrm{~mL}$ conical flasks and solvent S1 or S2 was added. Sonication was performed in ultrasonic bath of Supersonic X-3 Model DSD80A5QS instrument (power $80 \mathrm{Watt}$ ) at ambient temperature. Total time of sonication was $15 \mathrm{~min}$ for all samples.

Vortex mixing. Samples along with solvent S1 or S2 were placed in $50 \mathrm{~mL}$ conical flasks. Flasks were fitted in vortex mixture (manufactured by PCSIR, KarachiPakistan) and mixing was allowed for $15 \mathrm{~min}$ at ambient temperature.

Liquid chromatographic and mass spectrometric analyses. The LC-MS system used for analysis was consisted of FINNIGUN SURVEYOR units. PDA plus detector for UV detection, Auto-sampler plus injections of samples, LC pump plus for pumping mobile phase and LCQ advantage MAX for mass fragmentation of eluting compounds. X-Caliber 2.0 software programme was used for peak identification and peak integration. For the separation of both isomers, a reverse phase column was used with packing of ODS (Thermo Gold) due to its ability to retain polar compounds. The column was fitted in column oven and temperature of auto sampler as well as oven was kept ambient. Both of the standards ( $1 \& 2$ ) were detected in PDA detector at 280 $\mathrm{nm}$ but calculations were made on the basis of total ion chromatogram (TIC) obtained through MS detection. Mobile phase [acetonitrile:methanol 3:7 (A) and 0.1\% formic acid (B)] was run in gradient programme by starting at $5 \%$ B to reach $25 \%$ at $7 \mathrm{~min}$ and kept same for $2 \mathrm{~min}$; then $95 \%$ of $\mathrm{B}$ in two min followed by equilibration time of $4 \mathrm{~min}$.

Method validation. Sensitivity. Sensitivity of method was determined by limit of detection (LOD) and limit of quantification (LOQ). LOD and LOQ were calculated by using following equation:

$$
\mathrm{C}=\mathrm{K} \sigma / \text { Slope }
$$

Where:

$\mathrm{K}$ is 3 and 10 for LOD and LOQ, respectively; standard deviation (s) of response (at the retention time of analytes) of blank sample and slope values were obtained from the equations of straight line constructed by calibration standards. 
Specificity. Results of both standards and samples obtained by LC-MS analysis were compared regarding their retention times, UV absorption and mass spectra. The peak purity was assessed by checking UV absorption and mass spectrum of each peak from its start to end. Using X-Caliber software, mass of each peak was extracted by drawing a layout and putting $\mathrm{M}^{+1}$ value (291 of 1 and 2) in mass range. Only two peaks for these two isomers were observed in the total ion chromatogram (TIC) chart (Fig. 1).

Accuracy. For accuracy measurement, spiked samples were run along with standard 2 at different concentrations $(1.5,6.25$ and $25 \mathrm{mg} / \mathrm{L})$. The addition of the standard 2 into samples was also made prior to all extraction methods (section 2.3) from the plant material for analysis in order to check the interference of 2 with 1 .

Precision. Intraday and inter-day precision was carried out by running standard of varying concentration (i.e. $1.5-25 \mathrm{mg} / \mathrm{L}$ ) seven times in a day and with a gap of seven day, respectively. Retention time and TIC area of peak were taken into account in calculations and the results were taken in \% RSD.

DPPH assay. The DPPH assay was performed for the evaluation of antioxidant potential of plant extract as well pure compound (Molyneux 2004). The assay was performed by a reported method (Seeram et al., 2006) with slight modifications. Briefly, $1 \mathrm{~mL}$ of BHA or plant extract or pure reference standard ( 1 or 2 ) was added into $3 \mathrm{~mL}$ of DPPH solution in methanol $(12 \times 10$ $5 \mathrm{M})$. To calculate the time effect, absorption of mixtures was measured at $515 \mathrm{~nm}$ with 2 min time intervals until reaction reached to a stable level. The \% inhibition of DPPH by extract or pure compound was calculated by the following equation:

$$
\% \mathrm{DPPH}=\left[\mathrm{DPPH}_{\mathrm{A}}-\mathrm{DPPH}_{\mathrm{B}} / \mathrm{DPPH}_{\mathrm{A}}\right] \times 100
$$

Where:

$\mathrm{DPPH}_{\mathrm{A}}$ is initial absorption and DPPHB is the absorption after addition of standard $\left(\mathrm{BH}_{\mathrm{A}}\right)$ or plant samples or pure compounds 1 and 2 .

To calculate the IC50 values calibration curve was prepared for inhibition of DPPH by BHT and a linear equation was generated to calculate the inhibition of DPPH by the sample to its half. Concentration of BHT was taken in $\mu$ moles/L while samples were taken as $\mathrm{mg} / \mathrm{L}$ (known on the basis of LC-MS analysis).

\section{Results and Discussion}

Before developing the chromatographic separation method, three different extraction methods have been followed as described in experimental part.

Extraction techniques. Three different methods of extraction (sonication, microwave and vortex mixing,) with two solvent systems S1 and S2 (0.12 M HCl either in methanol:water or ethanol:water system) were adopted for the analysis of catechin in real samples of roots and aerial parts of $A$. camelorum. In all of these techniques, 15 min time was enough to extract the plant material. Longer time does not have any impact on recovery with any of the solvent system. The recovery of (1) was different from roots and aerial parts by applying different extraction techniques. The maximum recovery of (1) from aerial parts was found by microwave technique; while from the roots, vortex mixing as well as sonication was found to be best technique for maximum recovery (Table 1). These results are in agreement with previous findings by Quan et al. (2006).

Determination of catechins in $\boldsymbol{A}$. camelorum. The activities (e.g., food efficiency stimulation, antioxidant etc.) as well as external effects e.g., infections (Bandoniene and Murkovic, 2002) are associated with variation of concentration of both (1) and (2). Besides this, the difference in bioavailability of these both isomers also matters (Ghassempour et al., 2011). Thus, due to immense importance of (1) and (2) in pharmaceutical industry, a reliable chromatographic method was necessary to be developed, which may overcome the fatigues of previously reported methods such as complex mobile phase systems with higher flow rates (Donovan et al., 2006), longer time of analysis

Table 1. Catechins content in sample of A. camelorum

\begin{tabular}{lll}
\hline \hline Sample & $\mathrm{mg} / 100 \mathrm{~g}$ \\
\hline A-Son-S1 & 4.34 & \pm 0.05 \\
A-Son- S2 & 7.06 & \pm 0.06 \\
A-MW- S1 & 7.90 & \pm 0.06 \\
A-MW- S2 & $10.46 \pm 0.05$ \\
A-Vor- S2 & 8.49 & \pm 0.04 \\
A-Vor- S1 & $8.76 \quad \pm 0.06$ \\
R-Son- S1 & $9.43 \pm 0.05$ \\
R-Son- S2 & $10.02 \pm 0.03$ \\
R-MW- S2 & $9.12 \pm 0.08$ \\
R-MW- S1 & $9.87 \pm 0.08$ \\
R-Vor- S2 & $10.33 \pm 0.06$ \\
R-Vor- S1 & $9.33 \pm 0.02$ \\
\hline \hline
\end{tabular}



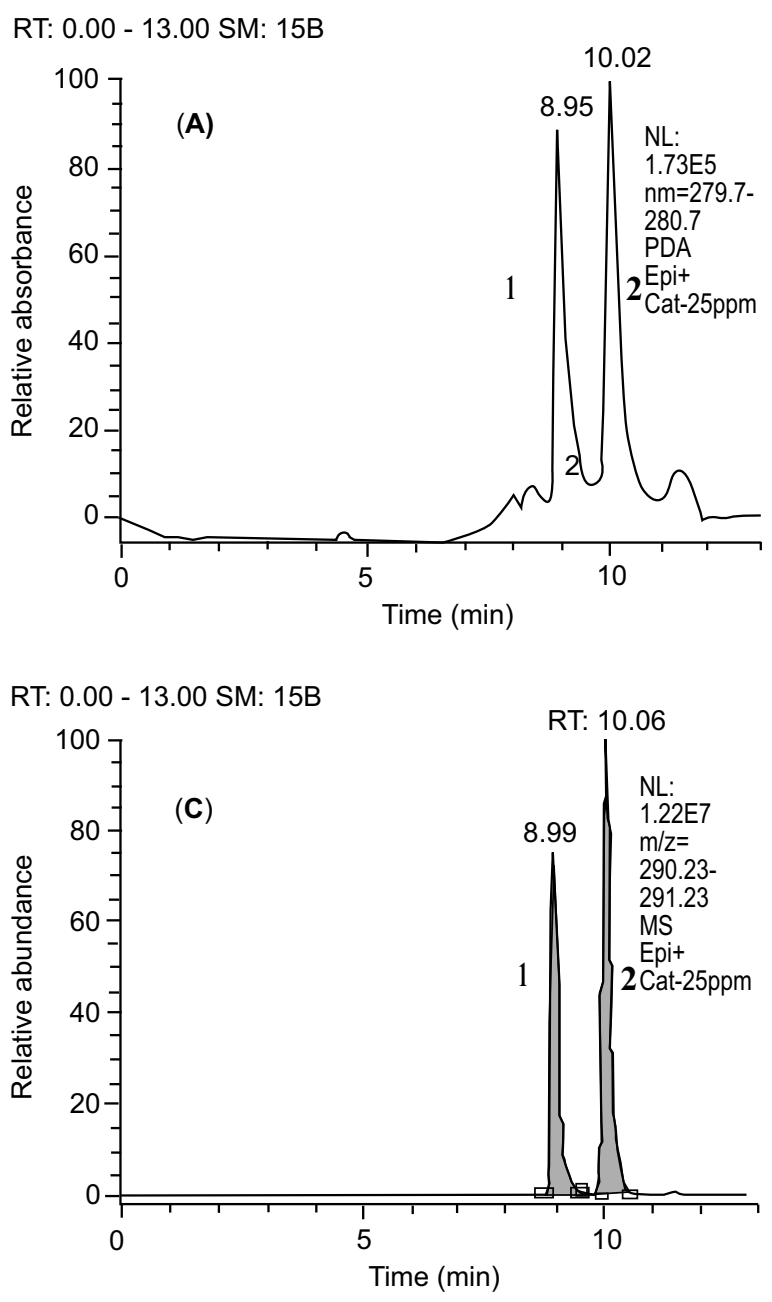

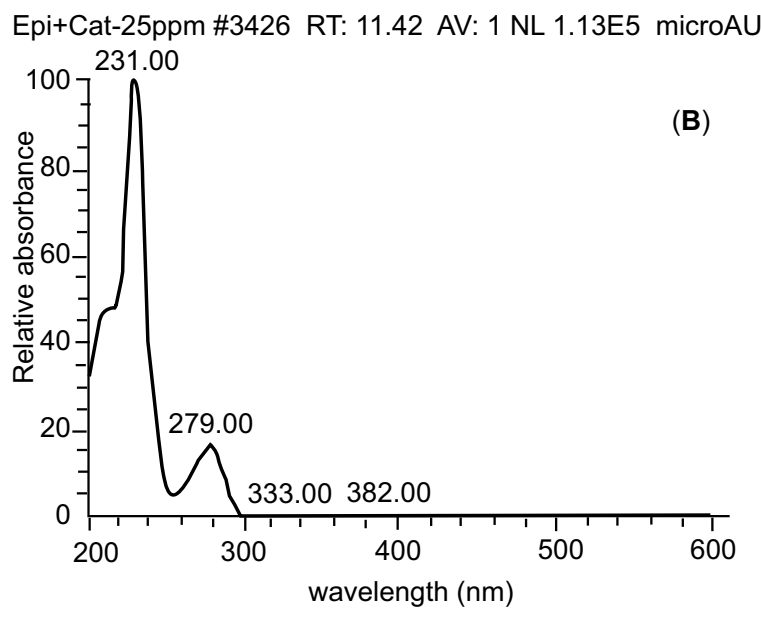

Epi+Cat-25ppm \#1203 RT: 10.02 AV: 1 SB: 733 030-6.39 NL T: +c APCl corona Full ms [200.00-350.00]

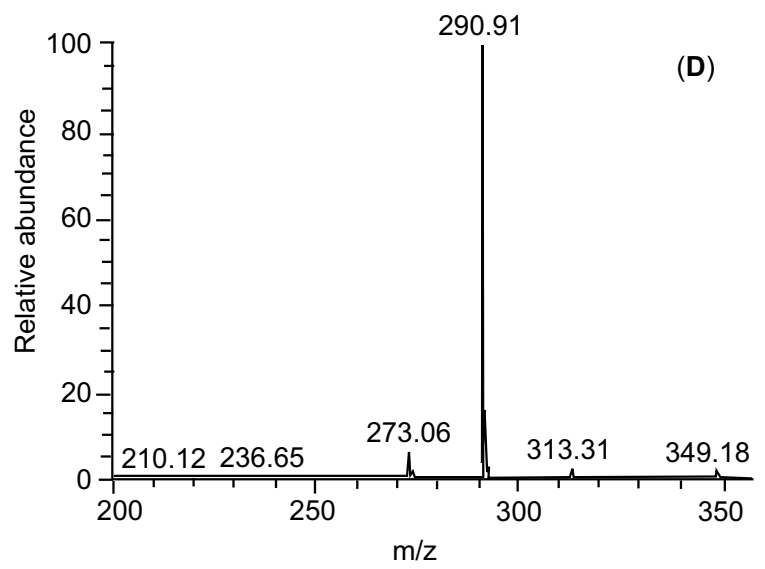

Fig.1. LC-MS profile of pure reference standards 1 and $\mathbf{2}$. $A=P D A$ chromatogram; $B=U V$ spectra; $C=$ total ion chromatogram; $\mathrm{D}=$ Mass spectrum (for 1 and 2).

(Dias et al., 2010) and higher detection limits (Soares et al., 2004; Viñas et al., 2000).

Separation of both isomers (1) and (2) (Fig. 1) in standard samples was performed by high-performance liquid chromatography (HPLC), whereas (1) was detected, confirmed and quantified (Table 1) in plant samples on the basis of both HPLC and total ion chromatogram (TIC) of mass spectrometry. In initial trials, concentration of solvent B was kept constant and solvent A was altered by changing concentration of acetonitrile. When isomers got separated with reasonable resolution; then mobile phase was selected as mentioned in experimental section. To have best resolution in short time, gradient programming was altered by changing percentage of solvent A from 95 to 25 . Going below $25 \%$ concentration of solvent $\mathrm{A}$, increased the retention time with some better resolution; while going above $25 \%$ concentration of A decreased the resolution. Therefore, programming of gradient was optimized at $25 \%$ concentration of A. Because (1) and (2) have similar response in all detection systems therefore; their separation is necessary even if someone has to determine one of these two isomers. The chance of false positive and false negative results may come out if one of them is taken as reference compound and determined in any sample. To avoid such calamity, proposed method is fair enough to separate both isomers and any one of these two can accurately be determined in any real sample. For verification, real samples were run accordingly by this method and found that $A$. camelorum contains only (1) as previously reported by Teissedre and Landrault (2000). The real samples of $A$. camelorum and standard sample of (1) 
RT: 0.00-13.00 SM: 15B
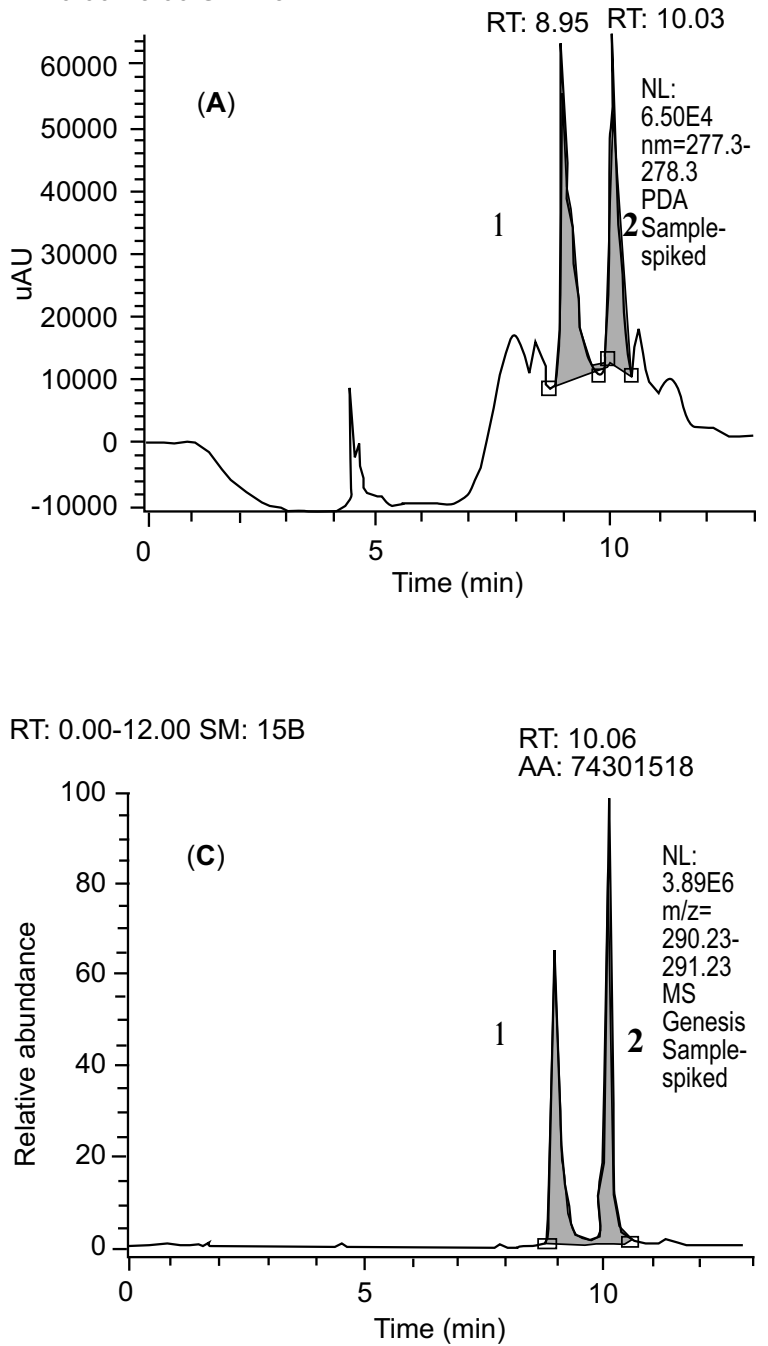

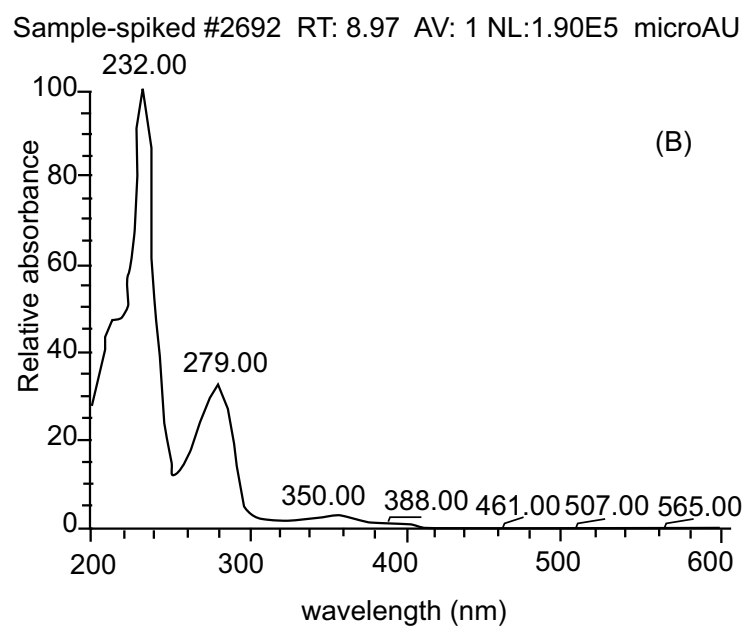

Sample-spiked \#1203 RT: 10.01 AV: 1 SB: 734 0.30-6.39 NL T: +c APCI corona Full ms [200.00-350.00]

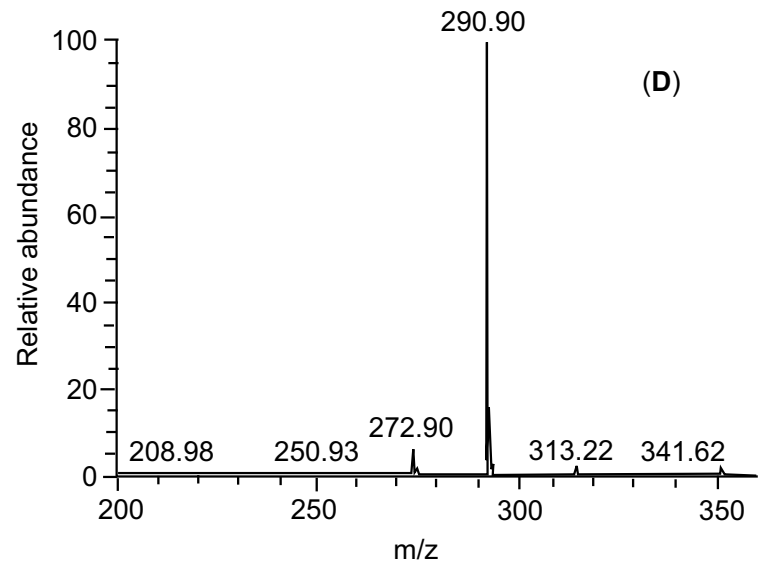

Fig. 2. LC-MS profile of sample. 1 is naturally present and spiked by adding $2 ; . A=P D A$ chromatogram; $B=$ UV spectra; $\mathrm{C}=$ Total Ion Chromatogram; $\mathrm{D}=$ Mass spectrum (for $1 \& 2$ ).

were spiked with the standard sample of (2); results showed that neither sample matrix nor 1 had interference with (2) (Fig. 2). It verified that proposed method may be a better choice for the analysis of (1) and (2), where accurate determination of either of these both isomers is required.
Validation of analytical method. For quantification of (1) and (2) in A. camelorum LC-MS method was validated. The concentration of reference standard was in the range of $1.5-25 \mathrm{mg} / \mathrm{L}$ having linear calibration curve with $\mathrm{r} 2>0.99$. LOD was $0.001 \mathrm{mg} / \mathrm{L}$ for both (1) and (2), while LOQ was $0.003 \mathrm{mg} / \mathrm{L}$ for (1) and (2),

Table 2. Validation data using MS detection

\begin{tabular}{llllllllll}
\hline \hline Compound & $\begin{array}{l}\text { Molecular } \\
\text { weight }\end{array}$ & $\mathrm{M}^{+1}$ & Intercept & Slope & $\begin{array}{l}\text { Correlation } \\
\text { coefficient }\end{array}$ & $\begin{array}{l}\text { Linearity } \\
\text { interval } \\
(\mathrm{mg} / \mathrm{L})\end{array}$ & RSD (\%) & $\begin{array}{l}\text { Detection } \\
\text { limit } \\
(\mathrm{mg} / \mathrm{L})\end{array}$ & $\begin{array}{l}\text { Quantific- } \\
\text { ation limit } \\
(\mathrm{mg} / \mathrm{L})\end{array}$ \\
\hline 1 & 290 & 291 & $4 \times 106$ & 617959 & 0.9994 & $1.5-25$ & 0.2 & 0.001 & 0.003 \\
2 & 290 & 291 & $6 \times 106$ & $8 \times 106$ & 0.9987 & $1.5-25$ & 0.9 & 0.001 & 0.003 \\
\hline \hline
\end{tabular}


Table 3. Antioxidant capacity of BHA, reference standards and samples measured by DPPH free radical assay

\begin{tabular}{lll}
\hline \hline Compound/Sample & $* \mathrm{IC}_{50}$ & \\
\hline BHA & 5.00 & \pm 0.2 \\
1 & 7.25 & \pm 0.3 \\
2 & 7.54 & \pm 0.3 \\
A-Son-S1 & 5.80 & \pm 0.3 \\
A-Son- S2 & 3.71 & \pm 0.2 \\
A-MW- S1 & 2.80 & \pm 0.0 \\
A-MW- S2 & 0.60 & \pm 0.0 \\
A-Vor- S2 & 2.40 & \pm 0.1 \\
A-Vor- S1 & 2.00 & \pm 0.1 \\
R-Son- S1 & 1.60 & \pm 0.1 \\
R-Son- S2 & 1.00 & \pm 0.1 \\
R-MW- S2 & 1.00 & \pm 0.0 \\
R-MW- S1 & 1.20 & \pm 0.1 \\
R-Vor- S2 & 0.70 & \pm 0.0 \\
R-Vor- S1 & 1.90 & \pm 0.1 \\
\hline \hline
\end{tabular}

*Concentration of BHA, reference compounds/samples (mg/L).

respectively. Data obtained during method validation studies are summarized in Table 2.

DPPH scavenging capacity. Antioxidant capacities of plant extract, pure antioxidant standards and pure compounds (1) and (2) were evaluated. All plant extracts were capable of scavenging the free DPPH radical. The $\mathrm{IC}_{50}$ i.e., the amount of plant extracts, needed to inhibit the DPPH activity by $50 \%$, was considered as measure of the antiradical capacity of plant extracts. The results of DPPH assay performed to calculate antiradical activities are listed in Table 3. Highest potential of free radical scavenging was shown by the extract from root sample prepared in solvent $\mathrm{S} 2$ by vortex mixing; while the extract from aerial parts prepared in S2 by microwave extraction method was found to be a potent radical scavenger. It has also been noticed that there is a close correlation between the amount of (1) present in the extracts of $A$. camelorum and the DPPH capacity as shown in Table 1-3. The plant extract samples with higher yield of (1) show greater DPPH capacity as compared to those with lower yield. This trend is in agreement with the reported literature (Molyneux, 2004). It may be due to the antioxidant nature of (1) present in higher amounts in the samples.

\section{Conclusion}

The present study demonstrates a validated analytical method for quantitative determination of catrchin (1) and epicatechin (2) in plant samples. Initially, optimization of instrumental parameters was achieved by different trials and then the method has been applied on real samples of $A$. camelorum. For extraction optimization, six samples from aerial and root parts of the plant were prepared with different extraction solvent systems and techniques and it has been observed that the high yield of (1) could be obtained by microwave extraction in S2 from aerial parts and by vortex mixing in $\mathrm{S} 1$ from root parts of A. camelorum. Besides this, free radical scavenging capacity of the $A$. camelorum extracts has also been determined by DPPH assay showing a close correlation with the amount of (1). From the results, it has been concluded that the developed method for the determination of (1) and (2) in the real plant extracts is more convenient, less time consuming, reliable and reproducible in terms of RSD, LOD and LOQ. Due to its simplicity and shorter time of analysis with more accuracy, the method could be a better choice for routine analytical measurements in pharmaceutical industries or in quality control laboratories for accurate determination of catechin or epicatechin when one or both are present in samples.

\section{Acknowledgement}

Support during this work by the PCSIR Laboratories Complex Karachi,Pakistan as well as National Centre of Excellence in Analytical Chemistry, University of Sindh, Jamshoro,Pakistan and H. E. J. Research Institute of Chemistry, International Center for Chemical and Biological Sciences, University of Karachi, Karachi,Pakistan is acknowledged. The authors are also thankful to Prof. Dr. Tahir Rajpoot, Director of Institute of Plant Science, University of Sindh for identification of plant.

\section{References}

Auger, C., Al-Awwadi, N., Bornet, A., Rouanet, J.M., Gasc, F., Cros, G., Teissedre, P.-L. 2004. Catechins and procyanidins in Mediterranean diets. Food Research International, 37: 233-245.

Bandoniene, D., Murkovic, M. 2002. On-line HPLCDPPH screening method for evaluation of radical scavenging phenols extracted from apples (Malus domestica L.). Journal of Agricultural and Food Chemistry, 50: 2482-2487.

Chang, W.C., Hsu, F.-L. 1989. Inhibition of platelet aggregation and arachidonate metabolism in platelets by procyanidins. Prostaglandins, Leukotrienes \& Essential Fatty Acids PLEFA, 38: 181-188. 
Crespy, V., Morand, C., Besson, C., Cotelle, N., Vézin, H., Demigné, C., Rémésy, C. 2003. The splanchnic metabolism of flavonoids highly differed according to the nature of the compound. American Journal of Physiology-Gastrointestinal and Liver Physiology, 284: G980-G988.

Dias, S. F., Lovillo, M. P., Barroso, C. G., David, J. M. 2010. Optimization and validation of a method for the direct determination of catechin and epicatechin in red wines by HPLC/fluorescence. Microchemical Journal, 96: 17-20.

Donovan, J. L., Crespy, V., Oliveira, M., Cooper, K. A., Gibson, B. B., Williamson, G. 2006. (+)Catechin is more bioavailable than (-)-catechin: relevance to the bioavailability of catechin from cocoa. Free Radical Research, 40: 1029-1034.

Donovan, J. L., Crespy, V., Manach, C., Morand, C., Besson, C., Scalbert, A., Rémésy, C. 2001. Catechin is metabolized by both the small intestine and liver of rats. The Journal of Nutrition, 131: 1753-1757.

Gali, H., Perchellet, E., Gao, X., Karchesy, J., Perchellet, J. 1994. Comparison of the inhibitory effects of monomeric, dimeric, and trimeric procyanidins on the biochemical markers of skin tumor promotion in mouse epidermis in vivo. Planta Medica, 60: 235-239.

Ghassempour, A., Mollayi, S., Farzaneh, M., SharifiTehrani, A., Aboul-Enein, H. Y. 2011. Variation of catechin, epicatechin and their enantiomers concentrations before and after wheat cultivarPuccinia triticina infection. Food Chemistry, 125: 1287-1290.

Iacopini, P., Baldi, M., Storchi, P., Sebastiani, L. 2008. Catechin, epicatechin, quercetin, rutin and resveratrol in red grape: Content, in vitro antioxidant activity and interactions. Journal of Food Composition and Analysis, 21: 589-598.

Ihm, S.H., Lee, J.O., Kim, S.-J., Seung, K.B., SchiniKerth, V. B., Chang, K., Oak, M.-H. 2009. Catechin prevents endothelial dysfunction in the prediabetic stage of OLETF rats by reducing vascular NADPH oxidase activity and expression. Atherosclerosis, 206: 47-53.

Marashdah, M., Al-Hazimi, H. 2010. Pharmacological activity of ethanolic extract of Alhagi maurorum roots. Arabian Journal of Chemistry, 3: 39-42.

Mendoza-Wilson, A. M., Glossman-Mitnik, D. 2006. Theoretical study of the molecular properties and chemical reactivity of (+)-catechin and (-)epicatechin related to their antioxidant ability.
Journal of Molecular Structure: THEOCHEM, 761: 97-106.

Molyneux, P. 2004. The use of the stable free radical diphenyl picryl hydrazyl (DPPH) for estimating antioxidant activity. Songklanakarin Journal Science Technology, 26: 211-219.

Naseri, M.K.G., Mard, S.A. 2007. Gastroprotective effect of Alhagi maurorum on exprimental gastric ulcer in rats. Pakistan Journal of Medical Science, 23: 570-573.

Okabe, S., Ochiai, Y., Aida, M., Park, K., Kim, S. J., Nomura, T., Suganuma, M., Fujiki, H. 1999. Mechanistic aspects of green tea as a cancer preventive: effect of components on human stomach cancer cell lines. Japanese Journal of Cancer Research, 90: 733-739.

Quan, P. T., Hang T. V., Ha, N. H., De, N. X., Tuyen, T. N. 2006. Microwave-Assisted Extraction of polyphenols from fresh tea shoot. Science and Technology Development, 9: 69-75.

Seeram, N. P., Lee, R., Scheuller, H. S., Heber, D. 2006. Identification of phenolic compounds in strawberries by liquid chromatography electrospray ionization mass spectroscopy. Food Chemistry, 97: 1-11.

Shaker, E., Mahmoud, H., Mnaa, S. 2010. Antiinflammatory and anti-ulcer activity of the extract from Alhagi maurorum (camelthorn). Food and Chemical Toxicology, 48: 2785-2790.

Silberberg, M., Morand, C., Manach, C., Scalbert, A., Remesy, C. 2005. Co-administration of quercetin and catechin in rats alters their absorption but not their metabolism. Life Sciences, 77: 3156-3167.

Soares, L., Oliveira, A., Ortega, G. G., Petrovick, P. 2004. Development and validation of a LC-method for determination of catechin and epicatechin in aqueous extractives from leaves of Maytenus ilicifolia. Journal of Pharmaceutical and Biomedical Analysis, 36: 787-790.

Sohn, O., Surace, A., Fiala, E., Richie, J., Colosimo, S., Zang, E., Weisburger, J. 1994. Effects of green and black tea on hepatic xenobiotic metabolizing systems in the male F344 rat. Xenobiotica, 24: 119127.

Teissedre, P.L., Landrault, N. 2000. Wine phenolics: contribution to dietary intake and bioavailability. Food Research International, 33: 461-467.

Terao, J., Piskula, M., Yao, Q. 1994. Protective effect of epicatechin, epicatechin gallate, and quercetin on lipid peroxidation in phospholipid bilayers. Archives of Biochemistry and Biophysics, 308: 
278-284.

Vennat, B., Pourrat, A., Pourrat, H., Gross, D., Bastide, P., Bastide, J. 1988. Procyanidins from the roots of Fragaria vesca: Characterization and pharmacological approach. Chemical and Pharmaceutical Bulletin, 36: 828-833.

Vinas, P., Lopez-Erroz, C., Marin-Hernandez, J. J.,
Hernandez-Cordoba, M. 2000. Determination of phenols in wines by liquid chromatography with photodiode array and fluorescence detection. Journal of Chromatography A, 871: 85-93.

Wolfe, K., Wu, X., Liu, R. H. 2003. Antioxidant activity of apple peels. Journal of Agricultural and Food Chemistry, 51: 609-614. 